\title{
Spontaneous otoacoustic emission recordings during contralateral pure-tone activation of medial olivocochlear reflex
}

\author{
E Bulut ${ }^{1,2}, L$ Öztürk ${ }^{2}$ \\ ${ }^{1}$ Department of Audiology, Trakya University Faculty of Health Sciences, Edirne, Turkey \\ ${ }^{2}$ Department of Physiology, Faculty of Medicine, Trakya University, Edirne, Turkey \\ Received: February 11, 2016 \\ Accepted: May 31, 2017
}

\begin{abstract}
We hypothesized that cochlear frequency discrimination occurs through medial olivocochlear efferent (MOCE)induced alterations in outer hair cell (OHC) electromotility, which is independent from basilar membrane traveling waves. After obtaining informed consent, volunteers with normal hearing ( $n=10$; mean age: $20.6 \pm 1.2$ years) and patients with unilateral deafness $(n=10$; mean age: $30.2 \pm 17.9$ years $)$ or bilateral deafness $(n=8$; mean age: $30.7 \pm 13.8$ years) underwent a complete physical and audiological examination, and audiological tests including transient evoked otoacoustic emission and spontaneous otoacoustic emission (TEOAE and SOAE, respectively). SOAE recordings were performed during contralateral pure-tone stimuli at 1 and $3 \mathrm{kHz}$. SOAE recordings in the presence of contralateral puretone stimuli showed frequency-specific activation out of the initial frequency range of SOAE responses. Basilar membrane motion during pure-tone stimulation results from $\mathrm{OHC}$ activation by means of MOCE neurons rather than from a traveling wave. Eventually, frequency-specific responses obtained from SOAEs suggested that OHC electromotility may be responsible for frequency discrimination of the cochlea independently from basilar membrane motion.

Keywords: otoacoustic emissions, spontaneous, hair cells, auditory, outer, cochlea, audiometry, pure-tone, medial olivocochlear efferents
\end{abstract}

\section{Introduction}

The separation of sound into component frequencies in the cochlea is thought to arise from the mechanical properties of the basilar membrane (2). Within the cochlea, the hydromechanical stimulus of sound leads to wave motions of the basilar membrane. These so-called "traveling waves" cause deflection of stereocilia on the apices of the hair cells. Deflection of a stereocilia bundle opens mechanically gated ion channels that allow potassium influx down through an electrochemical gradient from the potassium-rich endolymph fluid to cytoplasm of hair cell (14). Flow of potassium ions results in depolarization of the hair cell, which in turn stimulates afferent auditory nerve endings forming synapses with the basolateral aspect of the hair cell. A sound stimulus excites cochlear sensory epithelium by two mechanical forces: the pressure difference through the organ of Corti and the forces produced by contractility of outer hair cells (OHCs). Previous studies obtained important clues for the hypothesis that the mechanics of basilar membrane are determined by the intrinsic properties of $\mathrm{OHCs}$ (2). OHCs change their length in response to membrane potential alterations (21). OHC motility appears

\footnotetext{
Corresponding author: Erdogan Bulut, PhD

Department of Audiology, Trakya University Faculty of Health Sciences

Balkan Campus, Edirne 22030, Turkey

Phone: +90 28421330 42; Fax: +90 28421261 07; E-mail: erdoganbulut@gmail.com
} 
to actively amplify basilar membrane vibration, a process that is often referred to as cochlear amplification (2). OHCs are at the very center of cochlear amplification and frequency sensitivity $(13,17)$. A transmembrane motor protein called prestin was described in the lateral wall of OHCs. Prestin uses cytoplasmic anions as extrinsic voltage sensors and changes $\mathrm{OHC}$ length in response to membrane potential changes (11). In a recent study, prestin-based OHC motility was demonstrated to be necessary for mammalian cochlear amplification (12). Karavitaki and Mountain (22) elegantly demonstrated in gerbil cochlea that $\mathrm{OHC}$ contractions cause displacement of medial olivocochlear fibers and oscillatory fluid flow in the tunnel of Corti. They also suggested that such an OHC-driven fluid flow might be very important to cochlear function and hypothesized at the peak of the traveling wave, OHC contractions would push fluid into the tunnel of Corti, augmenting the original motion of the basilar membrane (22). Cochlear OHCs serve as both sensory receptors and biological motors (29). The contraction and relaxation properties of OHCs may play a significant role in discriminating the different frequency bands in the cochlea (22). The discovery of prestin supports this opinion $(11,12)$. The medial olivocochlear efferent (MOCE) branch synapses with OHCs, and the efferent pathway can be activated through electrical potential or a sound stimulus (18). Neurons of the MOCE system project to OHCs and convey signals that may control the sensitivity of the peripheral auditory system in a frequency-specific manner (9). Another type of hair cells, inner hair cells (IHCs), are considered to be stimulated by OHC-pushed fluid movement, which in turn leads to medial olivocochlear bundle induced inhibition of the first peak of the auditory nerve fiber response (19). IHCs are afferent transformers or primary receptors, whereas OHCs are efferent transformers or secondary receptors that obtain frequency selectivity.

Cochlear amplification of sound energy is evaluated by evoked otoacoustic emissions. Spontaneous otoacoustic emissions (SOAEs) are low-level acoustic signals measured in the human ear canal in the absence of any external stimulation. The presence of SOAEs suggests that cochlear hearing sensitivity is normal around the corresponding frequency band (5). A number of external conditions, such as pure-tones, ear canal pressure alterations, and contralateral acoustic stimuli affect SOAEs in various ways and are used for a better understanding of cochlear physiology. We studied the modification of SOAE recordings in the presence of contralateral pure-tone stimulation in humans. Interactions with pure-tones in the ipsilateral ear or contralateral stimulation with tonal or broadband signals were studied previously (10). In this study, we aimed to use pure-tones, i.e., 1 and $3 \mathrm{kHz}$, as contralateral acoustic stimuli. We hypothesized that efferent innervation of cochlea has a role in frequency discrimination function through $\mathrm{OHC}$ motility, rather than sound pressure-induced motility of basilar membrane.

\section{Methods}

\section{Subjects}

The study was designed and performed in accordance with the Declaration of Helsinki. After obtaining approval of the local ethical committee and informed consents, all volunteers underwent otoscopic examination, audiometry (subjective evaluation) and immitancemetry, and otoacoustic emissions (objective evaluation). Study group included individuals with normal hearing $(n=10 ; \mathrm{M} / \mathrm{F}, 4 / 6$; mean age $\pm \mathrm{SD}$ : $20.6 \pm 1.2$ years $)$, unilateral deafness $(n=10 ; \mathrm{M} / \mathrm{F}, 6 / 4$; mean age $\pm \mathrm{SD}: 30.2 \pm 17.9$ years $)$, and bilateral deafness $(n=8$; $\mathrm{M} / \mathrm{F}, 5 / 3$; mean age $\pm \mathrm{SD}: 30.7 \pm 13.8$ years). Exclusion criteria were tinnitus, middle-ear pathologies, or history of noise exposure and acoustic tumors. 
Audiometric tests

Hearing threshold assessment and low-frequency audiometry (Interacoustic Clinical Audiometers AC-40, Denmark) were performed in a sound-isolated chamber. For high frequencies, Koss R/80 (Koss Co., USA) earphones were used, whereas for low frequencies, TDH-39 (Telephonics, USA) earphones were preferred. Airway-hearing thresholds were determined by routine audiological analyses for values between 0.5 and $8 \mathrm{kHz}$ and by high frequencies of 8 and $10 \mathrm{kHz}$.

\section{Immittancemetric test}

Middle-ear pathologies and stapes reflexes were assessed by impedance audiometer (Interacoustic Audiometer AZ-7, Denmark) and a recording device (XYT Recorder AG-3, Denmark). The probe was set at $226 \mathrm{~Hz}$ and the pressure range of measurement was set at +200 to $-400 \mathrm{daPa}$. Type "A" tympanograms (peak pressure: between +50 and -100 $\mathrm{daPa}$ ) were accepted as normal.

\section{Transient evoked otoacoustic emission (TEOAE) recordings}

TEOAE tests were performed in a soundproof room using a Capella-Madsen adult OAE probe assembly (GN Otometrics A/S Taastrup, Denmark) fitted into the ear canal. The fastscreen menu option was used. Responses to clicks were windowed at 3-20 ms after stimulus onset and averaged following 2,080 repeated responses. The used stimulus was a non-linear, $40 \mu$ s click. Clicks were presented at $80 \mathrm{~dB}$ sound pressure level (SPL).

\section{Spontaneus otoacoustic emission recordings}

SOAEs were measured using Capella-Madsen adult OAE probe assembly (GN Otometrics A/S Taastrup, Denmark) fitted to the ear canal. Subjects were seated in a sound-isolated chamber. The equalized output of the system was flat $( \pm 5 \mathrm{~dB})$ from 0.5 to $10 \mathrm{kHz}$ with $12.7 \mathrm{~Hz}$ frequency resolution. The output from microphone was amplified. The response was subjected to spectral analysis. The SOAE sampling was based on averages of 500 accepted sweeps for the purpose of noise reduction. The grand average was calculated and the results were displayed. They were also characterized in terms of amplitude (dB SPL) and frequency $(\mathrm{Hz})$. SOAEs were visually identified as narrow peaks in the frequency spectrum and through a cursor function.

\section{Contralateral acoustic stimulus}

Contralateral acoustic stimuli (Interacoustic Diagnostic Audiometer AD-17, Denmark) were delivered through a small transducer connected to the appropriate ear canal through E-A-R Tone 3A insert earphones (Aearo Co., Indianapolis, IN, USA). Contralateral pure-tone stimulus intensities of 50 or $60 \mathrm{~dB}$ SPL during testing time at 1 or $3 \mathrm{kHz}$ were generated by the audiometer.

\section{Results}

\section{Hearing threshold}

In the control group, hearing threshold was within normal limits (i.e., below $20 \mathrm{~dB}$ HL) in all subjects. In the unilateral hearing loss group, hearing threshold was below $20 \mathrm{~dB} \mathrm{HL}$ at the intact side, whereas it was above $120 \mathrm{~dB}$ HL at the affected side in all patients. In the total hearing loss group, hearing threshold was equal to or above $120 \mathrm{~dB} \mathrm{HL}$ in all patients. 


\section{Stapes reflex threshold}

In the control group, all subjects showed intact stapes reflex activation at both sides between 80 and $110 \mathrm{~dB}$ SPL for 1 and $3 \mathrm{kHz}$ pure-tone stimuli. In the unilateral hearing loss group, stapes reflex was not generated at the affected side using ipsilateral 1 and $3 \mathrm{kHz}$ pure-tone stimulation between 80 and $110 \mathrm{~dB}$, whereas contralateral stimulation around 100 and 110 $\mathrm{dB}$ brought out stapes reflex. The stapes reflex threshold to ipsilateral 1 and $3 \mathrm{kHz}$ stimuli was also elevated to $100-110 \mathrm{~dB}$ in the intact side of patients with unilateral hearing loss. In the total hearing loss group, ipsilateral or contralateral 1 and $3 \mathrm{kHz}$ pure-tone acoustic stimuli between 80 and $110 \mathrm{~dB}$ SPL failed to activate stapes reflex in both ears. Stapes reflex threshold measurements are given in Supplementary Tables I-III.

\section{TEOAES}

To assess $\mathrm{OHC}$ activation, TEOAE reproducibility data were given as the mean \% value between 0.75 and $4 \mathrm{kHz}$. TEOAE responses in the control group showed that all participants had average response rate above $50 \%$, which verified that $\mathrm{OHC}$ activation was within physiological limits. TEOAE responses in the unilateral hearing loss group showed that these patients had average response rates above $50 \%$ in the intact side, whereas they had average response rates below $50 \%$ in the affected side. TEOAE responses in the total hearing loss group showed that all patients had average response rates below 50\%, which evidenced that OHCs of both ears were not functioning. TEOAE measurements are given in Supplementary Tables IV-VI.

\section{SOAES}

We performed SOAE recordings under two different conditions. The first condition included routine SOAE recording as described elsewhere (7), whereas the second condition included presence of a contralateral pure-tone acoustic stimulus. In the control group, when pure-tone stimulus was applied contralaterally below the threshold value of the stapes reflex, the recorded ear showed frequency-specific responses. These responses were recorded at puretone frequency bands and out of the first SOAE (without contralateral stimulus) responses in Fig. 1. Figure 1 demonstrates a group of representative sample recordings of SOAE either in the presence or absence of contralateral pure-tone stimuli ( 1 and $3 \mathrm{kHz}$ ). Contralateral $1 \mathrm{kHz}$, $50 \mathrm{~dB}$ sound stimulus led to activation around the $1 \mathrm{kHz}$ frequency band in SOAE. Similarly, $3 \mathrm{kHz}, 50 \mathrm{~dB}$ sound stimulus led to activation around the $3 \mathrm{kHz}$ frequency band. SOAE recordings either in the presence or absence of contralateral pure-tone (i.e., 1 and $3 \mathrm{kHz}$ ) stimuli were depicted in Fig. 2. In the absence of contralateral pure-tone stimulation, both graphics show activation around $9 \mathrm{kHz}$, which corresponds to the basal part of the cochlea. Interestingly, contralateral $1 \mathrm{kHz}$ pure-tone stimulus resulted in activation mostly around $1 \mathrm{kHz}$ frequency (Fig. 2A), whereas contralateral $3 \mathrm{kHz}$ pure-tone stimulus leads to an activation around $3 \mathrm{kHz}$ (Fig. 2B). In the total and unilateral hearing loss groups, we failed to observe any frequency-specific activation in the presence of contralateral pure-tone stimuli (Figs 3, 4, and 5). Interestingly, pure-tone acoustic stimuli applied to the intact ear did not lead to frequency-specific OHC activation in the affected side (Fig. 3). SOAE measurements are given in Supplementary Tables VII-IX.

\section{Discussion}

The main and novel finding of this study is that OHCs showed frequency-specific activity in the presence of pure-tone contralateral acoustic stimuli. OHCs may elongate and shorten in 

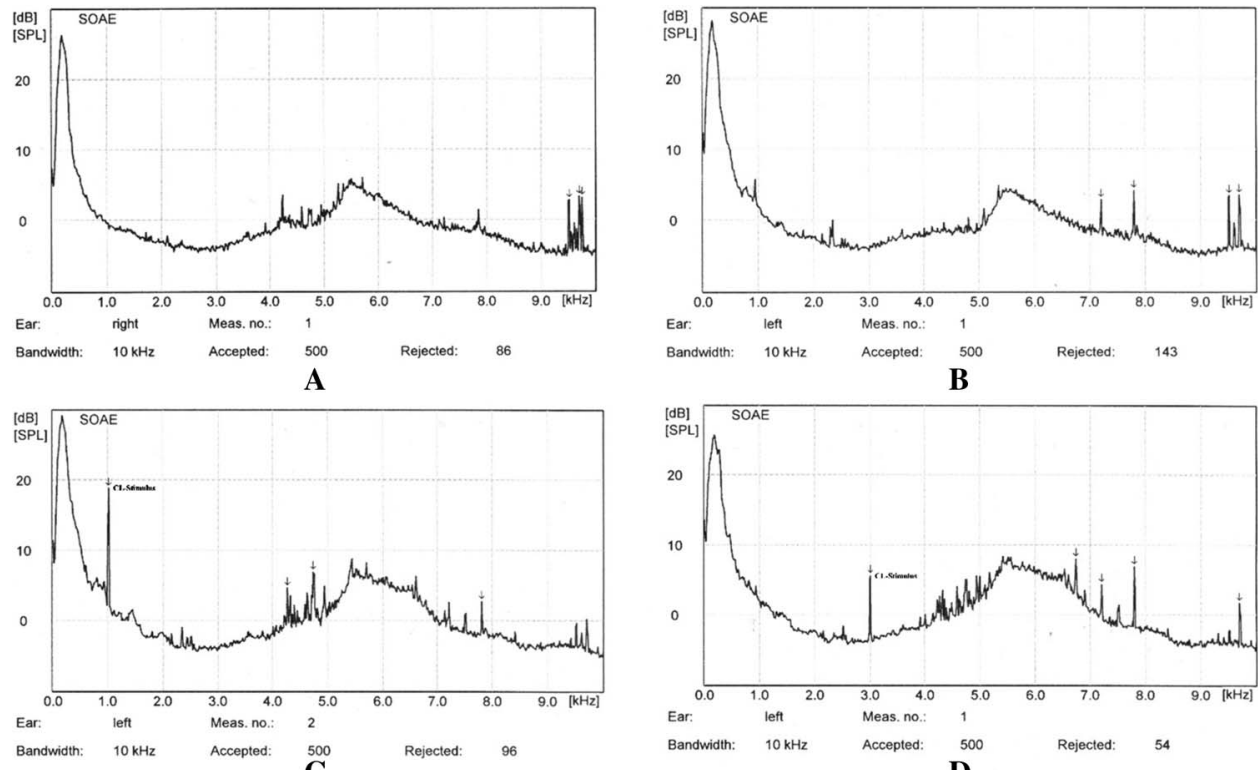

Fig. 1. Representative sample recordings of (A) SOAE right ear, (B) SOAE left ear, and (C and D) SOAE in the presence of contralateral pure-tone stimuli ( 1 and $3 \mathrm{kHz}$, respectively)

response to intracellular potential changes and these properties of $\mathrm{OHCs}$ are responsible for amplification of vibrations induced by a sound stimulus $(2,26)$. Active, non-linear mechanical processes present within the cochlea, interfered by the existence of OAE, stimulated further research into the function of the OHCs. The OHCs, with their efferent innervation, became the focus of attention for the source of mechanical energy. Isolated single OHCs have been demonstrated to contract as a result of electrical stimulation and altering the ionic environment of the bathing solution (4). Fast changes in OHC shape result from ATPindependent cellular electromotility (15) based on the activity of motor membrane protein prestin (33). Prestin is a direct electromechanical transducer providing the force required for cochlear amplification (12). The contraction and relaxation properties of OHCs may play a significant role in discriminating the different frequency bands in the cochlea (22). MOCE branch synapses with $\mathrm{OHCs}$, and the efferent pathway can be activated through electricity or a sound stimulus (18). The activation of these medial efferents can change OHC motile responses (9) and convert signals that are capable of controlling the sensitivity of the peripheral hearing system in a frequency-specific manner (18). IHCs, on the other hand, convey auditory information to the brain (11). It had been well established that the IHCs were detectors of movement in the organ of Corti, directly stimulating the auditory nerve (5). The IHCs are therefore considered to be primary "sensory" cells of the cochlea, whereas OHCs are termed "effectors," applying a mechanical input to basilar membrane motion. Terminals of olivocochlear bundle innervate OHCs and form a feedback system, which can modulate the gain of the cochlear amplifier (29). This suggested us that a frequency-specific reflex arc with its anatomical connections may exist between IHCs and OHCs. OHCs are targets of efferent innervation originating from the brainstem and activation of OHCs results in elevation of hearing threshold, i.e., a decrease in the systems' sensitivity. The main neurotransmitter of this efferent system is acetylcholine $(15,18)$. Isolated $\mathrm{OHC}$ recording 


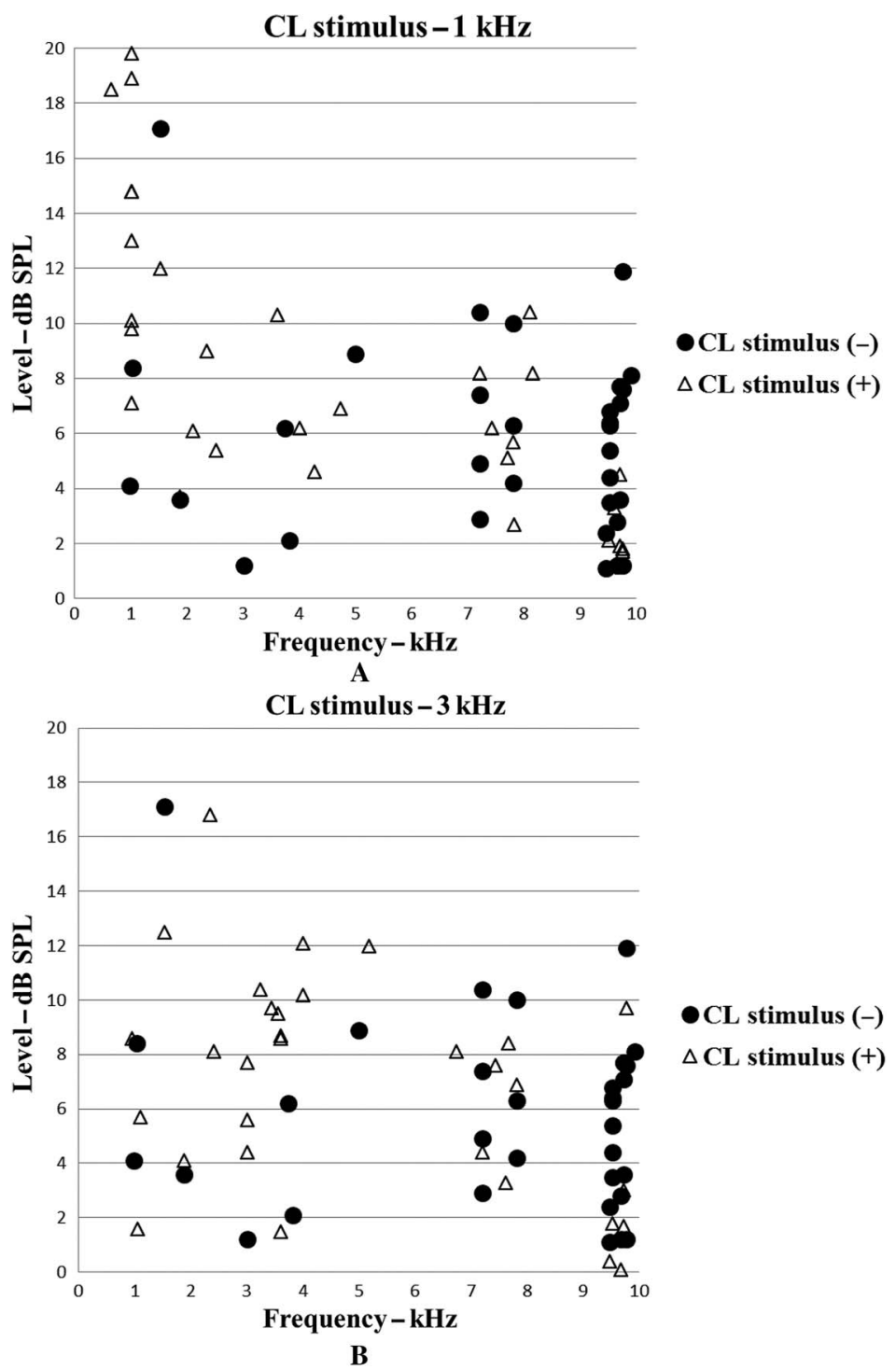

Fig. 2. SOAE recordings either in the presence or absence of a contralateral pure-tone [i.e., (A) 1 and

(B) $3 \mathrm{kHz}$ ] stimulus (normal hearing). In the absence of contralateral pure-tone stimulation, both graphics show an activation around $9 \mathrm{kHz}$ that corresponds to basal part of the cochlea. Interestingly, (A) contralateral $1 \mathrm{kHz}$ puretone stimulus resulted in an activation mostly around $1 \mathrm{kHz}$ frequency, whereas (B) contralateral $3 \mathrm{kHz}$ pure-tone stimulus led to an activation around $3 \mathrm{kHz}$

studies have provided functional evidence for cholinergic receptors localized around the base of the cell where the efferent synapses are located $(27,31)$. The olivocochlear bundle or the auditory efferent system originates in the brainstem and projects to the inner ear. Although the anatomy and physiology of the efferents are relatively well known, their functional roles in auditory perception remain to be elucidated (31). Initially, OHCs and IHCs were thought to differ in shape and number but to have similar functions. Studies on the sensory and supporting cells and on the innervation revealed that these two cell types differ dramatically. IHCs show primarily afferent innervation, whereas OHCs show efferent innervation. Olivocochlear bundle medial efferent branch makes synapses with $\mathrm{OHCs}$ and can be activated through electrical and sound stimuli $(5,10)$. Efferent innervation of OHCs seems 


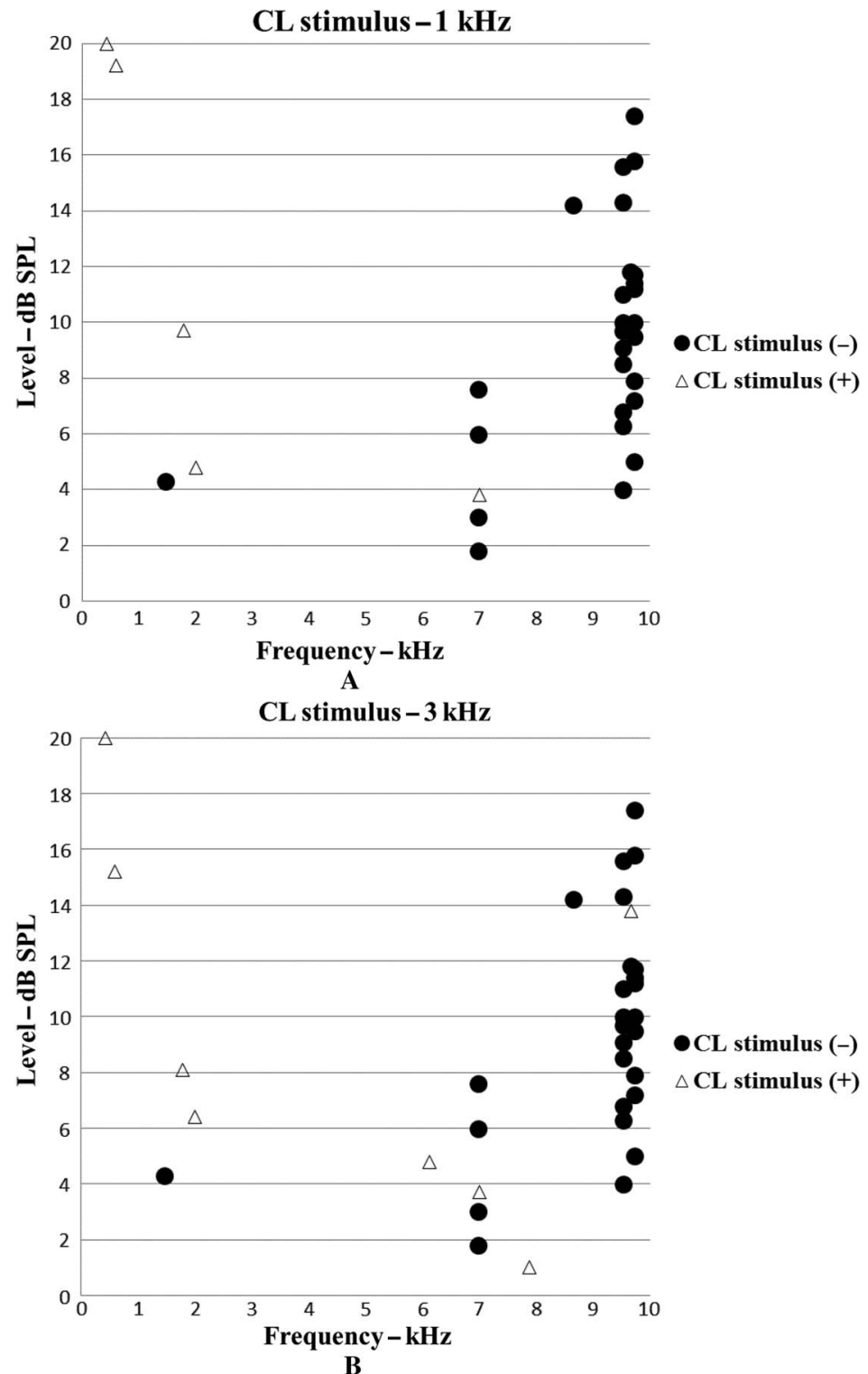

Fig. 3. SOAE recordings either in the presence or absence of a contralateral pure-tone [i.e., (A) 1 and

(B) $3 \mathrm{kHz}$ stimulus (unilateral hearing loss). Contralateral pure-tone stimulation was applied to the intact ear, neither of the graphics lead to an activation around 1 and $3 \mathrm{kHz}$ on the affected side

to modify the mechanical properties of the organ of Corti and basilar membrane $(9,27)$. This modification found in the organ of Corti is necessary for motion amplification at low SPLs (30). At different SPLs, basilar membrane vibrates in different frequency levels. At $30 \mathrm{~dB}$ SPLs, peak reticular lamina movements are twofold larger than those of the basilar membrane, a difference that becomes smaller as the stimulus level increases in the living cochlea. OHC motility is absent in postmortem period, which lacks cochlear amplification, and there is no phase or amplitude difference between the reticular lamina and basilar membrane in the postmortem period (32). 


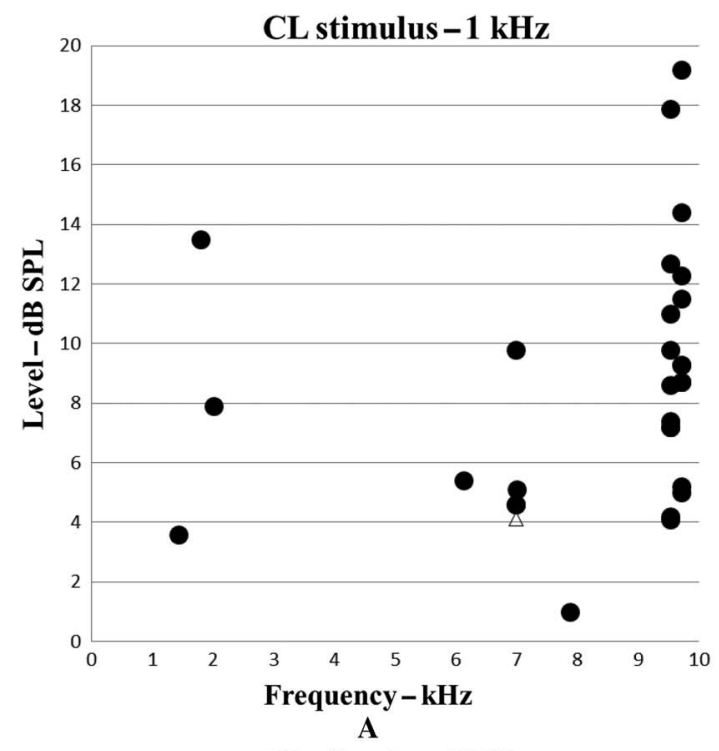

- CL stimulus (-)

$\triangle$ CL stimulus (+)

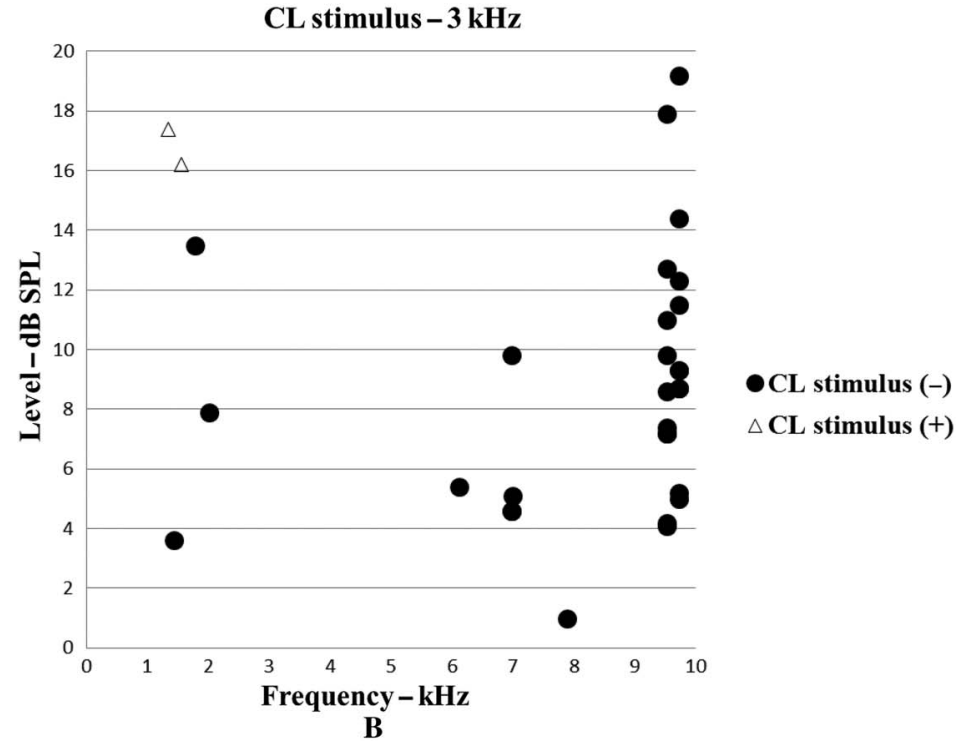

Fig. 4. SOAE recordings either in the presence or absence of a contralateral pure-tone [i.e., (A) 1 and

(B) $3 \mathrm{kHz}$ ] stimulus (unilateral hearing loss). Contralateral pure-tone stimulation was applied to affected side, both graphics did not lead to an activation around 1 and $3 \mathrm{kHz}$ in the intact ear

Pure-tone-induced frequency-specific activation in SOAE suggested a neural information transfer from one ear to the other. Furthermore, frequency discrimination may not be the result of basilar membrane vibrations, it may rather occur through a reflex pathway. In this study, we tried to obtain evidence for components of this hypothetic reflex arc: IHC as the receptive organ, connection from IHCs to superior olivary complex as the afferent way, synaptic connections in superior olivary complex as the center of reflex, connection from superior olivary complex to OHCs as the efferent way, and OHCs as the effector organs (6). This reflex activation occurs in all cochlear segments and uses IHCs, OHCs and the anatomic connections between them. Olivocochlear neural activation may play a key role in 


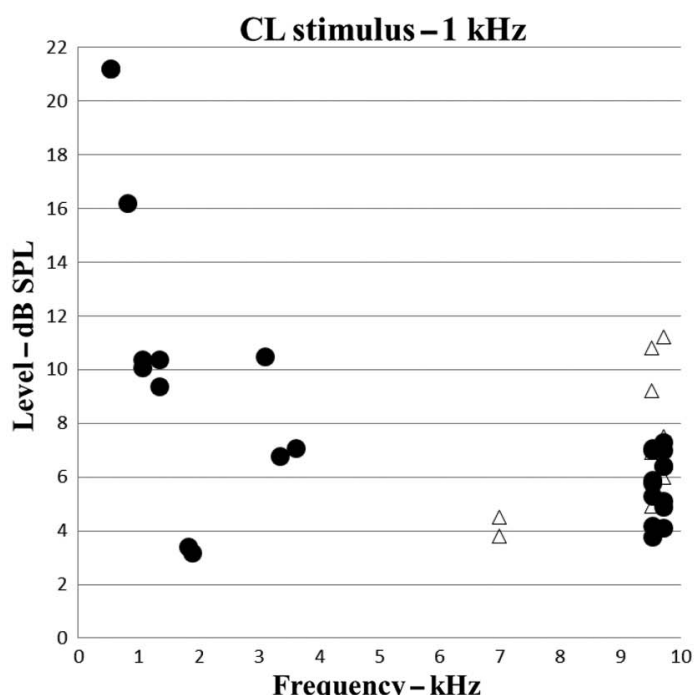

A

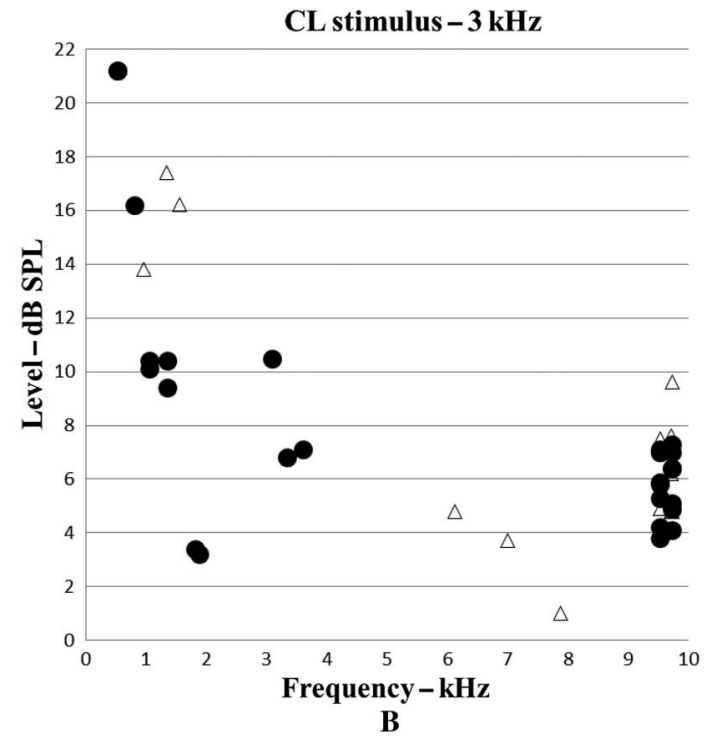

- CL stimulus (-)

$\triangle$ CL stimulus (+)

- CL stimulus (-)

$\triangle$ CL stimulus (+)
Fig. 5. SOAE recordings either in the presence or absence of a contralateral pure-tone [i.e., (A) 1,000 and (B) 3,000 Hz] stimulus (total hearing loss). In the presence of contralateral pure-tone stimulation, there was no activation around 1 and $3 \mathrm{kHz}$

this frequency discrimination with OHCs. Frequency-specific responses of OHCs suggest that the frequency information was received by the contralateral ear and then sent to the opposite ear. Therefore, OHCs in the ear that we recorded by SOAEs showed contractions in accordance with frequency information. Therefore, while initial SOAE recordings were around the frequency band of $9 \mathrm{kHz}$, they showed activity around the frequency band of corresponding stimuli in the presence of a contralateral pure-tone stimulus. We recorded $\mathrm{OHC}$ activity around $1 \mathrm{kHz}$, when the pure-tone stimulus was $1 \mathrm{kHz}$, and we recorded $\mathrm{OHC}$ activity around $3 \mathrm{kHz}$, when the pure-tone stimulus was $3 \mathrm{kHz}$. Previous studies demonstrated that contralateral sound suppressed both transiently evoked emissions (8) and distortion product emissions (23) in awake, human subjects. This suppression in response 
implies that the medial olivocochlear neurons alter the activity of the OHCs in such a way as to reduce the production of otoacoustic emissions. Recent studies of Berezina-Greene and Guinan (3) demonstrated that electrical excitation of MOCE fibers most often decreases stimulus-frequency otoacoustic emissions (SFOAEs), while it sometimes enhances SFOAEs in the frequency regions that are away from response dips. We demonstrated a suppression in the amplitude of spontaneous activity around $9 \mathrm{kHz}$, besides that activity, we also recorded frequency-specific activation, which was dependent on the frequency of contralateral puretone stimuli. The most noticeable changes in SOAEs are frequency shifts upward by $2-20 \mathrm{~Hz}$. It is widely believed that such changes occur as a result of stimulation of the crossed efferent pathways, which synapse directly on the OHCs (18). By using pure-tones and obtaining activations at the same frequency bands, we obtained strong evidence for such a crossed efferent pathway conveyance.

These frequency-specific responses were not due to the activation of an acoustic reflex arc, as the stimulus intensity was quite below the acoustic reflex threshold. Furthermore, in a previous study, Mott et al. (25) demonstrated that similar changes evoked by a contralateral tonal stimulus were not due to acoustic reflex activation. They measured contralateral acoustic reflex thresholds (CART) and observed longer latencies for supra-CART levels than for sub-CART levels; SOAE frequency and amplitude shifts observed during reflex activation conditions were larger than those observed during non-reflex conditions, and finally they measured reflex decay at $4,000 \mathrm{~Hz}$ and found that the reflex decayed $50 \%$ within $7 \mathrm{~s}$ and $100 \%$ within $20 \mathrm{~s}$. However, under prolonged contralateral acoustic stimulus conditions SOAE frequency was elevated for the entire $4 \mathrm{~min}$ of the stimulation period. In another study, sub-CART stimuli lead to the suppression of distortion product autoacoustic emission responses without activating an acoustic reflex and signal crossover (28). In this study, unilateral and bilateral hearing loss groups failed to show frequency-specific activation in SOAE, which provided another evidence for neural transfer of frequency information from one ear to the other. Otherwise, frequency-specific activity would be present in these groups.

In conclusion, a fast reflex-type pathway, not involving the higher centers of the brain, has already been suggested to be implicated in the suppression of transient emissions, by the onset of contralateral suppression taking around $20 \mathrm{~ms}$ to occur (20). Althen et al. (1) demonstrated in gerbil cochlea that frequency-specific regulation of the cochlear amplifier was mediated by MOCEs that were activated by contralateral acoustics stimulus. Recently, Lamas et al. (24) demonstrated that acoustic input and efferent activity regulate the expression of prestin at both transcriptional and post transcriptional levels. Another study showed that the OHC-type II spiral ganglion neurons drive the MOCE reflex-mediated control of the cochlear amplifier (16).

We obtained further evidence for such a neural pathway that conveys frequency information to the contralateral ear and evoke frequency-specific activation in the OHCs in the presence of contralateral pure-tone stimuli. We also suggest that frequency discrimination in all cochlear segments is a function of $\mathrm{OHC}$ electromotility stemming from efferent innervation.

\section{REFERENCES}

1. Althen H, Wittekindt A, Gaese B, Kössl M, Abel C: Effect of contralateral pure tone stimulation on distortion emissions suggests a frequency-specific functioning of the efferent cochlear control. J. Neurophysiol. 107, 19621969 (2012)

2. Ashmore JF, Mammano F: Can you still see the cochlea for the molecules? Curr. Opin. Neurobiol. 11, 449-454 (2001) 
3. Berezina-Greene MA, Guinan JJ Jr: Electrically evoked medial olivocochlear efferent effects on stimulus frequency otoacoustic emissions in guinea pigs. J. Assoc. Res. Otolaryngol. 18(1), 153-163 (2017)

4. Bray PJ (1989): Clicked evoked otoacoustic emissions and the development of a clinical otoacoustic hearing test instrument. $\mathrm{PhD}$ dissertation, London University, London, UK

5. Bright KE (2002): Spontaneous otoacoustic emissions. In: Otoacoustics Emissions: Clinical Applications (2nd ed.), eds Robinette MS, Glattke TJ, Thieme Medical Press, New York, pp. 77-94

6. Bulut E, Öztürk L (2006): The effect of basilar membrane on outer hair cells in frequency discrimination of the organ of Corti: a new hypothesis. In: Abstract Book of 32th National Congress of Turkish Society for Physiological Sciences, Denizli, Turkey, p. 82

7. Bulut E, Uzun C, Öztürk L, Turan P, Kanter M, Arbak S: A new hypothesis on the frequency discrimination of the cochlea. J. Int. Adv. Otol. (in press). doi:10.5152/iao.2017.2027

8. Collet L, Kemp DT, Veuillet E, Duclaux R, Moulin A, Morgon A: Effect of contralateral auditory stimuli on active cochlear micro-mechanical properties in human subjects. Hear. Res. 43, 251-262 (1990)

9. Cooper NP, Guinan JJ Jr: Efferent-mediated control of basilar membrane motion. J. Physiol. 576, 49-54 (2006)

10. Costa DL, Chibois A, Erre JP, Blanchet C, Charlet RS, Aran JM: Fast, slow, and steady-state effects of contralateral acoustic activation of the medial olivocochlear efferent system in awake guinea pigs: action of gentamicin. J. Neurophysiol. 78, 1826-1836 (1997)

11. Dallos P, Falkler B: Prestin, a new type of motor protein. Nat. Rev. Mol. Cell Biol. 3, 104-111 (2002)

12. Dallos P, Wu X, Cheatham MA, Gao J, Zheng J, Anderson CT, Jia S, Wang X, Cheng WH, Sengupta S, He DZ, Zuo J: Prestin-based outer hair cell motility is necessary for mammalian cochlear amplification. Neuron 58, 333-339 (2008)

13. Dong W, Olson ES: Detection of cochlear amplification and its activation. Biophys. J. 105, 1067-1078 (2013)

14. Friedman T, Battey J, Kachar B, Riazuddin S, Noben-Trauth K, Griffith A, Wilcox E: Modifier genes of hereditary hearing loss. Curr. Opin. Neurobiol. 10, 487-493 (2000)

15. Frolenkov GI, Mammano F, Kachar B: Regulation of outer hair cell cytoskeletal stiffness by intracellular $\mathrm{Ca}^{2+}$ : underlying mechanism and implications for cochlear mechanics. Cell Calcium 33, 185-195 (2003)

16. Froud KE, Wong AC, Cederholm JM, Klugmann M, Sandow SL, Julien JP, Ryan AF, Housley GD: Type II spiral ganglion afferent neurons drive medial olivocochlear reflex suppression of the cochlear amplifier. Nat. Commun. 6, 7115-7118 (2015)

17. Gao SS, Wang R, Raphael PD, Moayedi Y, Groves AK, Zuo J, Applegate BE, Oghalai JS: Vibration of the organ of Corti within the cochlear apex in mice. J. Neurophysiol. 112(5), 1192-1204 (2014)

18. Guinan JJ Jr: Olivocochlear efferents: anatomy, physiology, function, and the measurement of efferent effects in humans. Ear Hear. 27(6), 589-607 (2006)

19. Guinan JJ Jr, Lin T, Cheng H: Medial-olivocochlear efferent inhibition of the first peak of auditory-nerve responses: evidence for a new motion within the cochlea. J. Acoust. Soc. Am. 118, 2421-2433 (2005)

20. Hill JC, Prasher DK, Luxon LM: Evidence for efferent effects on auditory afferent activity, and their functional relevance. Clin. Otolaryngol. 22, 394-402 (1997)

21. Kachar B, Brownell R, Altschuler R, Fex J: Electrokinetic shape changes of cochlear outer hair cells. Nature 322 , 365-368 (1986)

22. Karavitaki KD, Mountain DC: Evidence for outer hair cell driven oscillatory fluid flow in the tunnel of Corti. Biophys. J. 92, 3284-3293 (2007)

23. Kujawa SG, Glattke TJ, Fallon M, Bobbin RP: Contralateral sound suppresses distortion product otoacoustic emissions through cholinergic mechanisms. Hear. Res. 68, 97-106 (1993)

24. Lamas V, Arévalo JC, Juiz JM, Merchán MA: Acoustic input and efferent activity regulate the expression of molecules involved in cochlear micromechanics. Front. Syst. Neurosci. 8, 253 (2015)

25. Mott JB, Norton SJ, Neely ST, Warr WB: Changes in spontaneous otoacoustic emissions produced by acoustic stimulation of the contralateral ear. Hear. Res. 38, 229-242 (1989)

26. Robles L, Ruggero MA: Mechanics of the mammalian cochlea. Physiol. Rev. 81, 1305-1352 (2001)

27. Slepeckly NB (1996): The cochlear structure. In: The Cochlea, eds Dallos P, Popper AN, Fay RR, Springer, New York, pp. 44-130

28. Sun XM: Contralateral suppression of distortion product otoacoustic emissions and the middle-ear muscle reflex in human ears. Hear. Res. 237, 66-75 (2008)

29. Thiers FA, Nadol JB, Liberman MC: Reciprocal synapses between outer hair cells and their afferent terminals: evidence for a local neural network in the mammalian cochlea. J. Assoc. Res. Otolaryngol. 9(4), 477-489 (2008)

30. Zagadou BF, Mountain DC: Analysis of the cochlear amplifier fluid pump hypothesis. JARO 13, 185-197 (2012) 
31. Zeng FG, Martino KM, Linthicum FH, Soli SD: Auditory perception in the vestibular neurectomy subjects. Hear. Res. 142, 102-112 (2000)

32. Zha D, Chen F, Ramamoorthy S, Fridberger A, Choudhury N, Jacques SL, Wang RK, Nuttall AL: In vivo outer hair cell length changes expose the active process in the cochlea. PLoS One 7(4), e32757 (2012)

33. Zheng J, Shen W, He DZ, Long KB, Madison LD, Dallos P: Prestin is the motor protein of cochlear outer hair cells. Nature 405, 149-155 (2000) 